\title{
Musculoskeletal power Doppler
}

\author{
J.M. Rubin
}

Department of Radiology, University of Michigan, 1500E Medical Center DR, Ann Arbor, MI 48109, USA

\section{Introduction}

Musculoskeletal applications of ultrasound imaging are one of the most rapidly growing areas of ultrasonography. Because they are superficial structures, muscles and tendons are almost perfect targets for ultrasound imaging. Furthermore, because inflammatory changes are frequently associated with increased blood flow, a sensitive blood-flow monitor, such as power Doppler, could be very useful in these cases, particularly since the grayscale changes associated with inflammation are non-specific. Recent literature appears to support this notion.

\section{Assessment of musculoskeletal inflammation}

In a study by Newman et al. [1] 23 patients with focal musculoskeletal symptoms were scanned in grayscale and power Doppler. Fourteen patients with shoulder pain made up the largest cohort. Increased blood flow subjectively by power Doppler was seen in 22 of the 23 patients (Fig.1). The single patient without increased flow had a normal sterile hip effusion. The conclusion was that power Doppler could offer additional information regarding inflammatory conditions particularly when grayscale is non-specific.

Similar techniques can be used for fluid collections [2]. It would be very helpful to know whether joint effusions or soft tissue fluid collections are inflamed or not. Such a distinction would be very useful. Four grades of blood flow were subjectively evaluated. Of the $36 \mathrm{col}-$ lections with moderate to increased blood flow, 35 had an inflammatory or neoplastic etiology. Of the seven effusions or fluid collections without significant increase in signal, there was no inflammatory component. Grayscale imaging proved neither sensitive nor specific for acute inflammation (sensitivity $79 \%$, specificity $62 \%$; Figs. 2, 3).
One issue that arose here, and needed to be addressed throughout all these studies, is the gain setting for the measurements. The classic teaching of setting the gain at the noise floor may not apply because of the strong bone surface specular reflector [3]. Phase jitter artifact which is enhanced in power Doppler, and the non-zero possibility that signal will appear in the noise, makes the spurious imaging of flow in the underlying bone possible. Because of this, the gain threshold is set such that there is no observed signal in the bone [3].

\section{Therapy monitoring}

Although it appears that power Doppler can preliminarily detect disease, an area of some interest would be the ability to monitor the progression of therapy. There is preliminary evidence that that is in fact the case [4]. In eight knees in seven patients with inflammatory arthritis with knee pain, there was a measurable decrease in synovial blood flow in seven knees after joint aspiration and steroid injection intra-articularly. These results corresponded very well with symptomatic relief. In five patients the contralateral asymptomatic knee showed no changes in interval scanning; thus, there seems to be the potential for power Doppler to monitor therapy of inflammatory arthritis (Fig.4).

\section{Other applications of musculoskeletal power Doppler}

Blood-flow monitoring in other soft tissue abnormalities has also been evaluated. In reflex sympathetic dystrophy of the lower extremity, a syndrome arising frequently following surgery or injury to a limb, there is a painful disorder of hyperesthesia, skin-color changes, local warmth, swelling, and decreased range of motion. In these cases power Doppler is able to distinguish flow changes in the dorsum of the foot [5]. In 30 patients and 26 asymptomatic controls, there was a significant increase in the abnormal vs the normal feet $(p<0.005)$. 
The side-by-side comparison of feet showed a trend, but no significant difference between sides $(p<0.20)$. Power Doppler has also proven useful in imaging for postoperative patency of vascular anastomoses in microsurgical tissue transfers [6].

The enthusiasm displayed in the aforementioned two studies has not held up uniformly. An issue of some importance is distinguishing transient synovitis from infectious hip effusions in children. The consequences of an incorrect diagnosis include permanent disability and a life-long hip deformity [7]. The clinical differentiation between the two conditions in young children can be difficult, and the diagnosis may require hip aspiration in all cases [8]. For the same reasons, the power Doppler detection of increased blood flow would serve as a useful adjunct for distinguishing infected from sterile effusions. Hopefully, the method would make tapping certain effusions unnecessary. Unfortunately, power Doppler did not prove sensitive enough to discriminate between infected and non-infected hips. All of the transient synovitis cases did not have increased flow compared with the opposite hip; however, only, at most, 3 of 11 proven cases of septic arthritis had increased flow compared with the opposite side [7].

There are still applications for power Doppler in pediatric hip imaging, however. There are definite reasons why imaging blood flow to the femoral head is desirable. A particularly valuable application is in monitoring blood flow in the severe abduction and flexion of the hip for therapy of hip dysplasias [9]. If the hip is abducted too far, blood flow can be cut off causing avascular necrosis of the femoral head. Power Doppler can be used to monitor flow in the medial circumflex artery and its branches. These are the main vascular supplies to the femoral head.

It was discovered that in 11 of 13 normal neonate studies, there was an abduction angle beyond which flow disappeared with loss of standard spectral Doppler signals. The angle varied between 60 and $85^{\circ}$, and flow returned immediately after decreasing the angle beneath that limit. The presumption is that if these patients had required therapy for hip dysplasia, they would have been at risk for avascular necrosis if abducted too far. In addition, the normal spectra in the femoral heads of neonates have now been characterized [10]. Unlike in adult limbs, the normal mean resistive index (RI) is $48 \%$, and hopefully this number could be used to evaluate external compression of the arterial supply based on increasing RI. The vessels were identified and documented using power Doppler.

\section{Blood-flow quantification}

More quantitative assessment of blood flow in extremities is also possible. With the use of fractional blood-volume estimates [11], estimates of the amount of moving blood in tissue before and after an intervention can be made. One application is to assess increases in blood flow after exercise (Fig. 5) [12]. The effectiveness of an exercise regimen in a quantitative way could then be evaluated. Ten volunteers performed a programmed series of curls with power Doppler scan before and after exercise. Fractional blood-volume estimates were also made. Qualitative assessments of increased blood flow after exercise were highly significant $(p<0.0005)$, and fractional blood-volume estimates were also significantly increased $(p<0.01)$ with a mean increase of $470 \%$.

In addition, we recently performed a preliminary study of skin heating in six normal volunteers in which skin temperature was elevated to 38,40 , and $42^{\circ} \mathrm{C}$ on three separate occasions in random sequences. The issue being tested was whether skin heating could induce increase blood flow in the underlying muscle. This suggests a physiologic basis for heat application as a means of relieving muscle pain by heating. A heating blanket set at the desired temperature was placed over the subject's upper back, and scanning was performed in power Doppler mode before, during, and $30 \mathrm{~min}$ after heating at the desired temperature. The operator was blinded to the applied temperature in each case. The blood flow in the underlying trapezius muscle was measured at 18 different sites, and compared with the preheating blood flow at the same sites. Using a general linearized statistical model without carry-over effects, there was a significant increase in the amount of flow in the trapezius muscle $(p<0.015)$. These preliminary data suggest that real blood-flow increases in underlying muscle do occur with skin heating at the aforementioned temperatures.

Fig. 1. Acute supraspinatis tendonitis with increased flow in tendon on power Doppler

Fig. 2. Lateral epicondyle of the left elbow in patient with acute gout. Marked inflammation is demonstrated on power Doppler

Fig.3a, b. Transverse scan of the dorsal left wrist showing a septic tenosynovitis secondary to staphylococcus. a Grayscale image shows an inflamed tendon with a hypoechoic cleft (arrow) through it and fluid around it. b Power Doppler image shows that the cleft and much of the presumed fluid actually represents enlarged blood vessels secondary to the inflammation

Fig. 4a, b. Left shoulder septic bursitis. a Initial ultrasound scan shows fluid $(F)$ in the bursa and large amount of flow on power Doppler. The white blood cell count was 100,000 . b Repeat scan 1 month later after surgical debridement and antibiotic therapy shows residual fluid $(F)$ with definitely less flow. Symptoms had improved

Fig. 5a, b. Muscle blood flow pre- and post exercise. a Pre-exercise scan shows blood flow in the biceps muscle. b Post-exercise scan shows dramatically increase, almost by a factor of four, in blood volume in the muscle. The settings in both scans are the same 

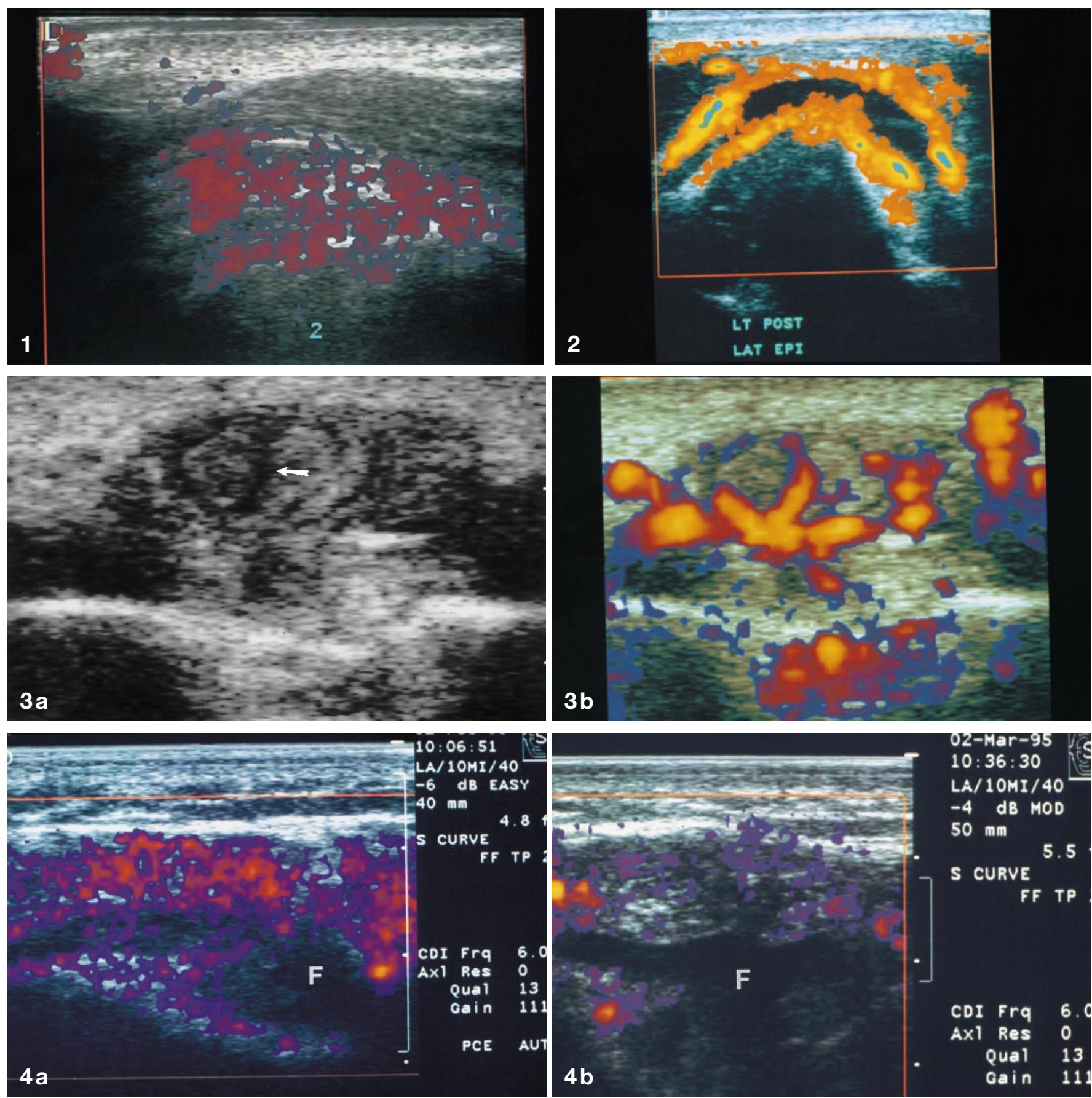

O2-Mar-95

$10: 36: 30$

LA/ $10 \mathrm{MI} / 40$

-4 dB MOD

$50 \mathrm{~mm}$

S CURVE

5.5

FF TP
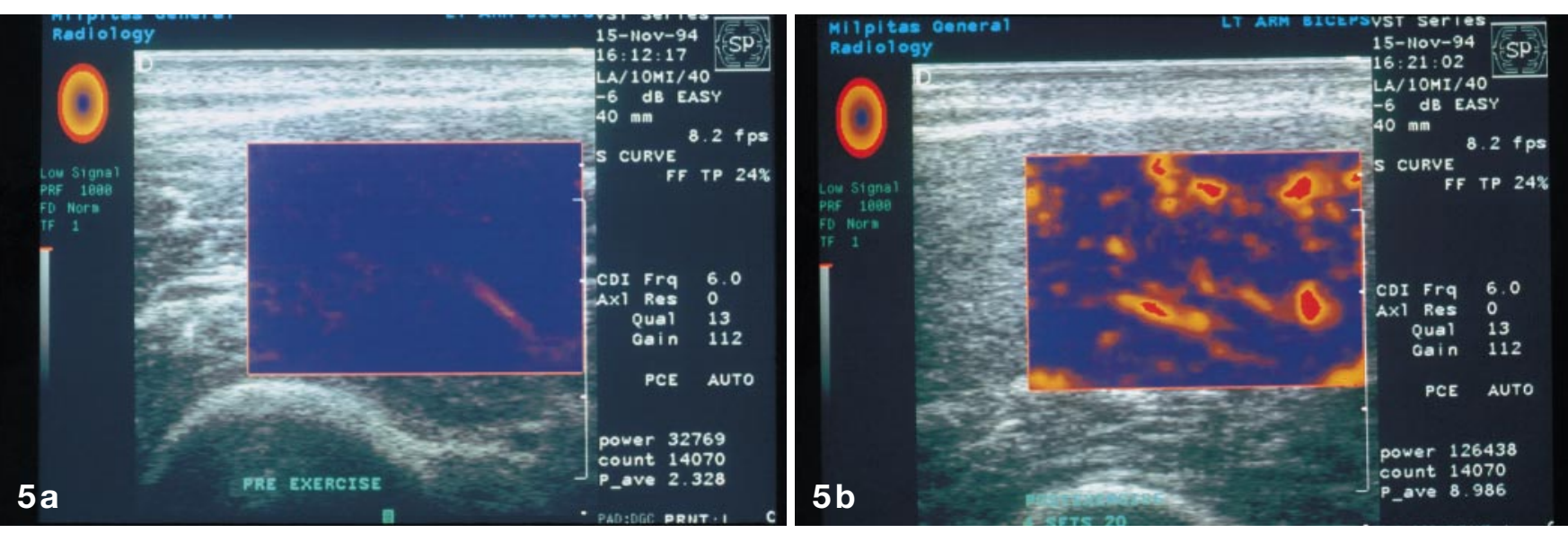

$\begin{aligned} \text { CDI Frq } & 6 . \\ \text { Axl Res } & 0 \\ \text { Qual } & 13 \\ \text { Gain } & 11\end{aligned}$




\section{References}

1. Newman JS, Adler RS, Bude RO, Rubin JM (1994) Detection of soft-tissue hyperemia: value of power Doppler sonography. AJR 163: 385-389

2. Breidahl WH, Newman JS, Taljanovic MS, Adler RS (1996) Power Doppler sonography in the assessment of muscloskeletal fluid collections. AJR 166: 1443-1446.

3. Rubin JM, Bude RO, Carson PL, Bree RL, Adler RS (1994) Power Doppler: a potentially useful alternative to mean-frequency based color Doppler sonography. Radiology 190: 853-856

4. Newman JS, Laing TJ, McCarthy CJ, Adler RS (1996) Power Doppler sonography of synovitis: assessment of therapeutic response: preliminary observations. Radiology 198: 582-584

5. Nazarian LN, Schweitzer ME, Mandel S et al. (1998) Increased soft-tissue blood flow in patients with reflex sympathetic dystrophy of the lower extremity revealed by power Doppler sonography. AJR 171: 1245-1250
6. Loh N, Ch'en IY, Olcott E et al. (1997) Power Doppler imaging in preoperative planning and postoperative monitoring of muscle flaps. J Clin Ultrasound 25: 465-471

7. Strouse PJ, DiPietro MA, Adler RS (1998) Pediatric hip effusions: evaluation with power Doppler sonography. Radiology 206: 731-735

8. Fink AM, Berman L, Edwards D, Jacobson SK (1995) The irritable hip: immediate ultrasound guided aspiration and prevention of hospital admission. Arch Dis Child 72: 110-114

9. Bearcroft PW, Berman LH, Robinson AHN, Butler GJ (1996) Vascularity of the neonatal femoral head: in vivo demonstration with power Doppler US. Radiology 200: 209-211

10. Schwartz DS, Keller MS, Fields JM, States LJ, Marsh JS (1998) Arterial waveforms in the femoral heads of healthy neonates. AJR 170: 465-466

11. Rubin JM, Adler RS, Fowlkes J et al. (1995) Fractional moving blood volume estimation using power Doppler imaging. Radiology 197: 183-190

12. Newman JS, Adler RS, Rubin JM (1997) Power Doppler sonography: use in measuring alterations in muscle blood volume after exercise. AJR 168: 1525-1530 